\title{
Structure of the Photon
}

\author{
J.M. Butterworth \\ Department of Physics and Astronomy \\ University College London London, WC1E 6BT
}

\section{Introduction}

\subsection{The Structure of a Fundamental Gauge Boson?}

The photon is of course the gauge boson of QED, and is as far as we know elementary. When we measure photon "structure", what we are in fact doing is probing the quantum fluctuations of the field theory.

The photon couples - via a splitting into virtual, charged fermion-antifermion pairs - into the electroweak and strong interactions. Such behaviour is an important aspect of quantum field theories, and similar phenomena arise in many different situations. For example, the gluon splitting to quarks drives the scaling violations in hadronic structure. Studies of photon structure test our understanding of this behaviour. In this talk I give an overview of the current data and phenomenology, and outline some opportunities available in the medium term future.

\subsection{How photon structure is measured}

The quick answer to the question "How do you measure the structure of the photon" is unfortunately "With great difficulty". Experiments measuring the photon structure in general use the almost on-shell photons accompanying $e^{+}$or $e^{-}$beams. These photons are typically probed by some short distance process. This may be deep inelastic scattering [1], high transverse energy $\left(E_{\mathrm{t}}\right)$ jets $[2,3,4,5,6]$ or particles or heavy quark production $[7,8]$.

These are in general rather challenging measurements. One key problem is the fact that the target photons have a spectrum of energies. If this is integrated over, sensitivity to the photon structure is lost. Thus, some way must be found to measure the photon energy on an event-by-event basis. Another area of difficulty is that although the presence of high $E_{\mathrm{t}}$ particles does imply the presence of a short distance scale, the exact relationship between the distance scale and $E_{\mathrm{t}}$ is not clear.

The leading order processes for jet, particle and heavy quark production are shown in figure 1 . In each case the virtual parton propagator probes photon at a scale related to $E_{\mathrm{t}}^{2}$. 
The diagrams in which the photon enters into the hard process directly ("direct processes") and those in which a partonic photon structure is resolved ("resolved processes") have comparable cross sections. At higher orders, and in real data, separation between resolved and direct is at some level arbitrary, since if the photon splits to a $q \bar{q}$ pair of virtuality $\mu$, the process could be categorized as either resolved or NLO direct, depending upon whether the factorization scale is chosen to be greater or less than $\mu$. Despite this, it is still possible and useful to select events in which a greater or lesser fraction of the photon's momentum enters into the hard process, where the hard process is defined in terms of observables such as jets. Such a selection is often made on the basis of the variable

$$
x_{\gamma}^{\mathrm{OBS}}=\frac{\sum E_{T}^{\mathrm{Jet}} e^{-\eta^{j e t}}}{2 y E_{e}}=\frac{\sum_{j e t s} E-p_{z}}{\left(E-p_{z}\right)_{\gamma}}
$$

which is the fraction the photon momentum entering into the jets [3]. Direct processes have high $x_{\gamma}^{\mathrm{OBS}}$ and resolved processes have low $x_{\gamma}^{\mathrm{OBS}}$. Since $x_{\gamma}^{\mathrm{OBS}}$ is a kinematic variable defined in terms of jets, it is calculable to any order in $\mathrm{QCD}$ and in any model desired.

The other major process by which photon structure is measured is deep inelastic $e \gamma$ scattering (also shown in figure 1). In this case an inclusive measurement of the cross section is made as a function of the four-momentum transfer at the lepton vertex $\left(Q^{2}\right)$ and/or the Bjorken scaling variables $x$ and $y$. This allows the extraction of structure functions, defined in exactly the same way as for a nucleon. Neglecting weak interactions (since for current experiments $\mathrm{Q}^{2} \ll M_{W}^{2}$ ), this gives:

$$
\frac{d^{2} \sigma_{e \gamma \rightarrow e X}}{d x d \mathrm{Q}^{2}}=\frac{2 \pi \alpha^{2}}{x Q^{4}}\left[\left(1+(1-y)^{2}\right) F_{2}^{\gamma}\left(x, \mathrm{Q}^{2}, \mathrm{P}^{2}\right)-y^{2} F_{L}^{\gamma}\left(x, \mathrm{Q}^{2}, \mathrm{P}^{2}\right)\right]
$$

where if $X=\mu^{+} \mu^{-}$the QED structure is being probed (i.e.the number of muons 'in' the photon) and if $X=$ hadrons the QCD structure is being probed (i.e.the number of quarks 'in' the photon). For DIS, $\mathrm{Q}^{2} \gg \mathrm{P}^{2}$, that is a "highly virtual" photon probes a less virtual photon at scale $\mathrm{Q}^{2}$.

\section{QED Structure Function}

The QED structure of the photon is exactly calculable, since no strong interaction is involved. The OPAL measurement is shown in figure 2. The data are in good agreement with fundamental prediction of QED (solid line). Measurements have also been made by the other LEP experiments [9]. Noteworthy features include the peak at high $x$ values, and the fact that the structure function increases with $\mathrm{Q}^{2}$. Both effects are due to the first splitting of the photon into $\mu^{+} \mu^{-}$, a least one of which carries a large fraction of the photon energy. As $\mathrm{Q}^{2}$ increases, smaller and smaller 


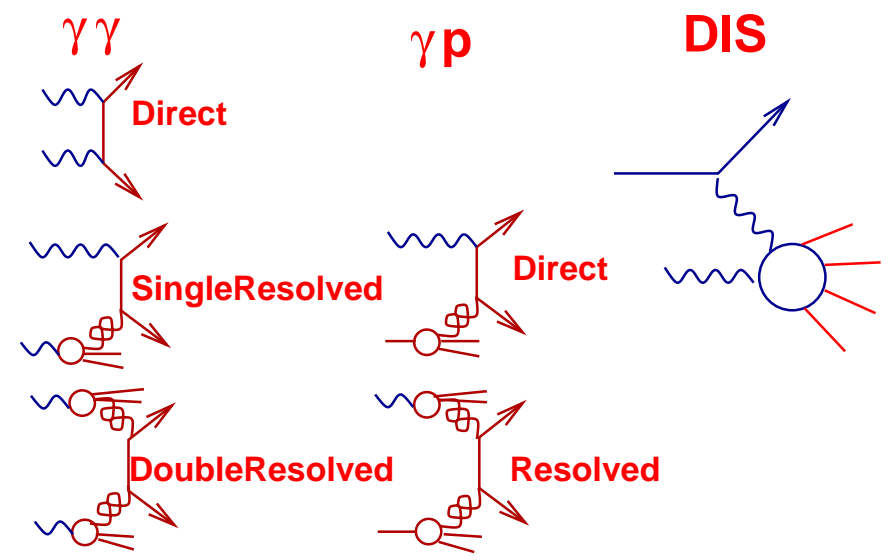

Figure 1: Examples of leading order diagrams for jets and heavy flavour production at HERA and LEP, and DIS at $e^{+} e^{-}$machines.

splittings of the photon can be resolved and thus the structure function increases, even at high $x$.

Before moving on to compare with the QCD structure, two other points should be made here. Firstly, the $x$ resolution is fairly good (around 0.03). This is because $W$, the $\mu^{+} \mu^{-}$mass, is well measured, and hence the target photon energy is well known. A second point is that the virtuality of the target photon has a significant effect for all $\mathrm{Q}^{2}$, even though $\left\langle\mathrm{P}^{2}\right\rangle=0.05 \mathrm{GeV}^{2}$. This can be seen by comparing the solid line, where the virtuality has been taken into account, with the dashed line, where the target photon has been assumed to be real.

\section{QCD and the 'Real' Photon}

The case in which the photon splits into a $q \bar{q}$ pair is theoretically more complex. If the quarks have a low transverse momentum $\left(k_{T}\right)$ with respect to each other, they can exist for times which are long on the time-scale of the the strong interaction. Thus, a complex partonic system can evolve, consisting of a mixture of perturbative and non-perturbative physics. In addition to the presence of non-perturbative physics in the initial state, the final state also involves long-distance hadronization processes.

The QCD structure of the photon is also experimentally more problematic. Jets of hadrons are in general harder to measure than muons. This does not just affect measurements of jet or particle production - where issues such as fragmentation and 'underlying events' become important. It is also an issue in DIS, since the photon energy must be measured from the final state. Thus it becomes critical to contain 


\section{OPAL}
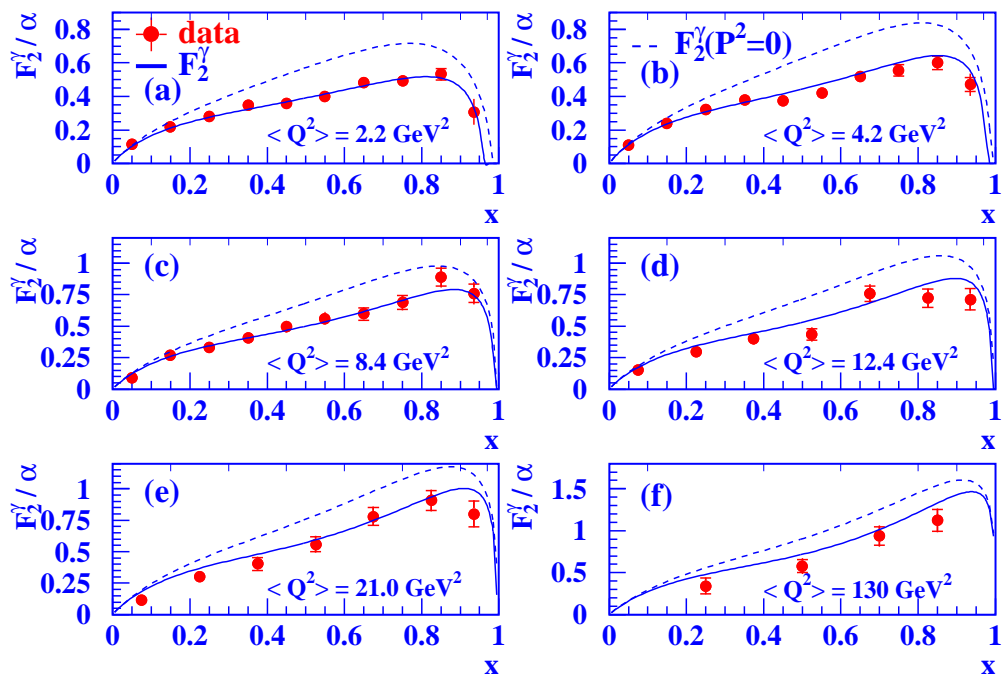

Figure 2: The QED structure function of the $F_{2}$ photon. The structure function is measured by the OPAL Collab. as a function of the Bjorken scaling variable $x$ in six different bins of the hard scale, $\mathrm{Q}^{2}$. Measurements have also been made by the other LEP collaborations [9].

as much of the hadronic system as possible in the detector, and vital to have the best possible estimates of the structure of this final state to allow estimates of the resolution and acceptance to be reliably made [10].

These effects mean that there is potentially large model dependence in any extraction of photon structure. Dealing with this model dependence is a major issue for the experiments. Best practice is to separate the measurement as much as possible from interpretation. Thus measurements of cross sections are made with the minimal model dependence. These cross sections are defined in terms of the real final state (hadrons rather than partons!) and the kinematic regions in which they are measured are dictated by detector acceptance. Because of this, they are unfortunately often hard to interpret and compare with each other or with fundamental QCD.

These cross sections are sensitive to many important effects, not only the photon structure, but that of the proton (in particular the gluons at $x \approx 10^{-2}$ at HERA), as well as $\alpha_{s}$, low- $x$ QCD, hadronization and "underlying" events. There is not much benefit in being sensitive to all these at the same time, and thus the role of phenomenology is critical to isolate as far as possible different effects. Several programs allowing flexible calculation of the processes in QCD at NLO are available [11, 12] and are crucial in the ongoing effort to extract fundamental physics from a wide range of data. Equally important are general purpose Monte Carlo simulations [13] 
which include models of hadronization and underlying events as well as (typically) LO QCD and parton showers. These allow us to build a consistent picture of these processes over a large data set from several experiments, and also allow improvements in our understanding to be fed back into modelling of detector acceptance, leading to improved measurements.
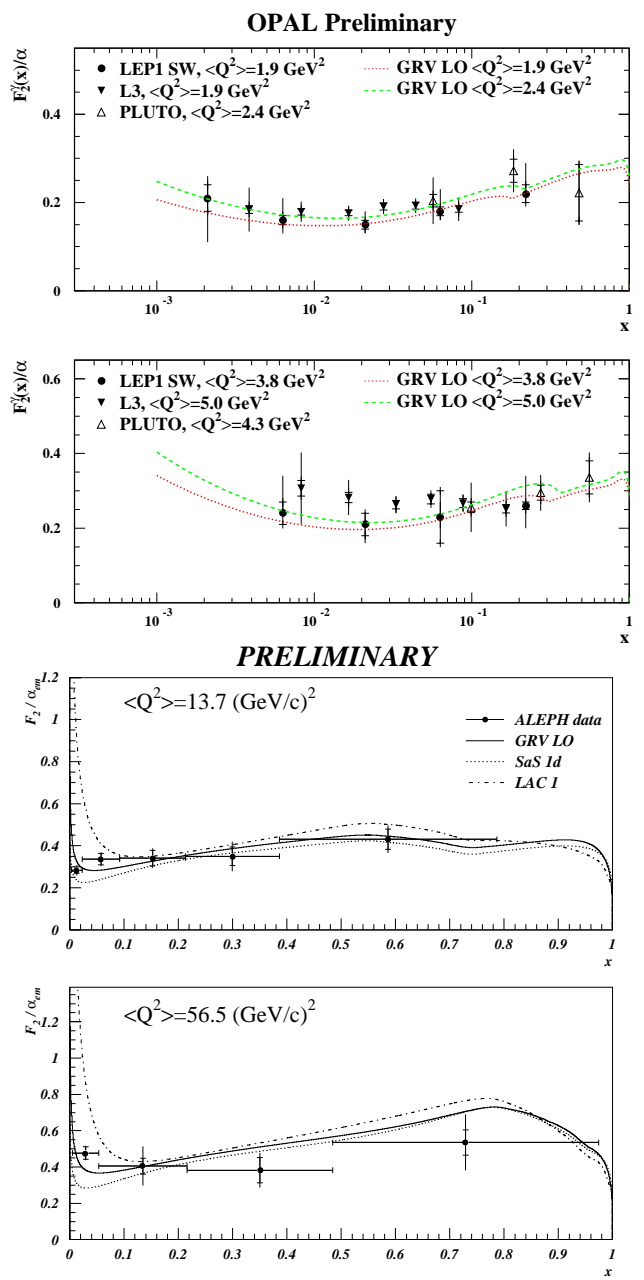

Figure 3: Examples of recent data on the QCD structure function $F_{2}$ of the photon (see text).

The potential rewards are very high, and these are exciting times for those involved. However, it is very much "work in progress", and the following represents a snapshot of an evolving field. See these [14] references for more details. 


\subsection{QCD Structure Function}

The QCD structure of the photon has been measured for samples with $0.24 \mathrm{GeV}^{2}<$ $\left\langle\mathrm{Q}^{2}\right\rangle<400 \mathrm{GeV}^{2}[1]$. An example of the more recent data at low $x$ and intermediate $\mathrm{Q}^{2}$ is shown in figure 3a, from OPAL and L3. Earlier PLUTO data are also shown. The data are compared to curves from the GRV group [15]. In the GRV model of the proton, the rise in $F_{2}^{\text {proton }}$ is generated by the DGLAP evolution [16]. The same behaviour is expected in the photon parton distributions at similar $x$, and can be seen in the curves. Unfortunately the data do not yet have the reach or accuracy to determine whether or not such a rise occurs. However, improved statistics and smaller systematic uncertainties are expected before the end of LEP running. It should be noted that L3 sometimes display their data without showing an estimate of the model dependence, preferring instead to show a series of data sets each corrected according to a different model. Here, these different data sets have been used to estimate the systematic uncertainty [17].

Figure $3 b$ shows preliminary measurements at a higher $\mathrm{Q}^{2}$ from ALEPH. Since these are plotted on a linear scale in $x$, they can easily be compared to the QED structure functions of figure 2. Whilst at low $x$ the gluon splitting means that the structure function is expected to rise like that of the proton, at higher $x$ the photon to two fermion splitting dominates and the behaviour is similar for the QED and QCD structure functions.

Also clearly seen in the data is the expected positive scaling violation at all $x$, driven by photon splitting at high $x$ and (like the proton) by the gluons at low $x$. The data are summarized in figure 4.

\subsection{QCD and the Real Photon at HERA}

Since at HERA the photon is probed by a parton from the proton, HERA does not measure $F_{2}^{\gamma}$. The HERA equivalent of $F_{2}^{\gamma}$ is a jet cross section. This has the major disadvantage that hadronization, as well as choice of jet definition, plays a role. An advantage, however, is that the gluon distribution in the photon enters directly in the cross section at leading order. A further advantage is that due to the fact that the $\mathrm{CM}$ frame is boosted strongly in the proton direction, the photon remnant tends to open out and be relatively well measured in the detector. In addition, both ZEUS and H1 have small angle taggers, which allow the photon energy to be inferred from the electron energy. These effects mean that the target photon energy is better measured than at LEP.

The measured cross sections may be compared to NLO pQCD calculations, which take a photon parton distribution function (PDF) as input. If the jets have high enough transverse energy $\left(E_{T}^{\mathrm{Jet}}\right)$ the hadronization corrections are expected to be at the level of a few percent. The probing scale is something of the order of $E_{T}^{\mathrm{Jet}}$. 


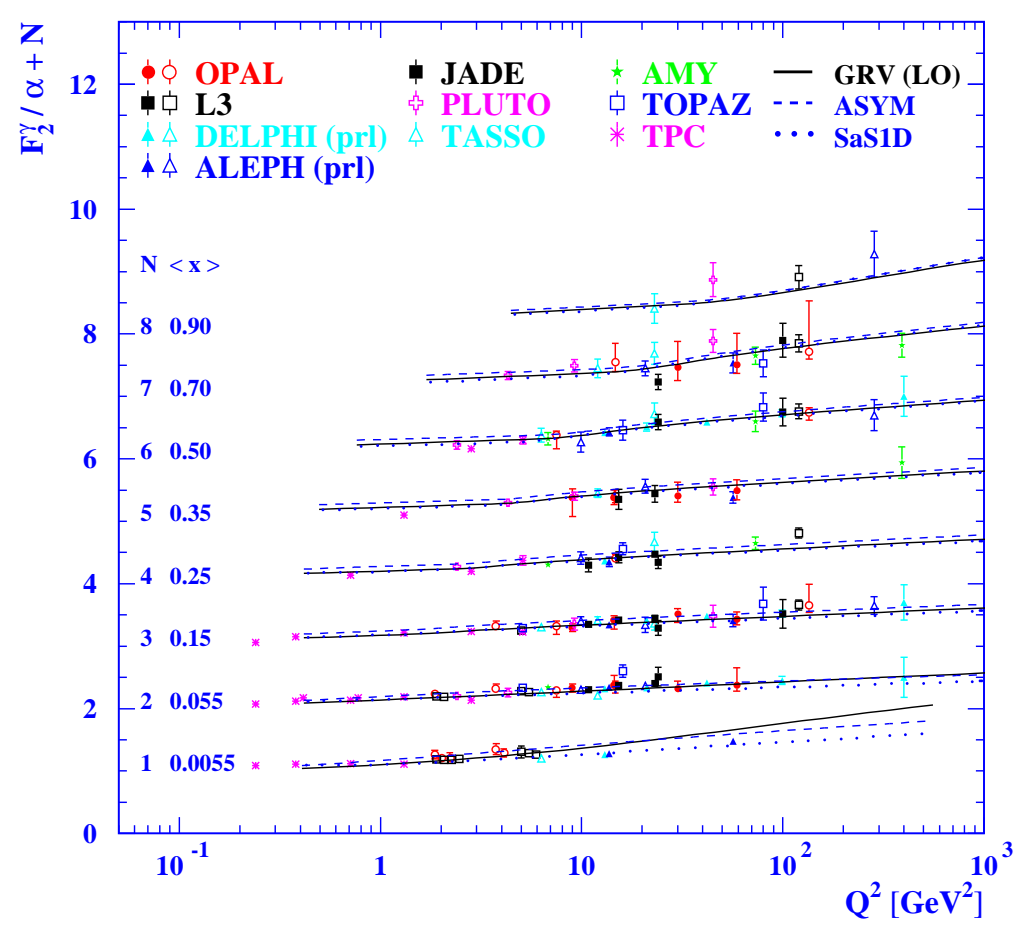

Figure 4: $\mathrm{Q}^{2}$ dependence of the QCD structure function.

The latest ZEUS preliminary data are shown in figure 5 for differential cross sections defined as in [5] but now measured above a variety of $E_{T}^{\text {Jet }}$ thresholds, increasing the hard scale. The data are compared to a calculation [11] using the AFG-HO Photon PDF [18]. The high $x_{\gamma}^{\mathrm{OBS}}$ data is in excellent agreement with the theory. However, in the region including both high and low $x_{\gamma}^{\mathrm{OBS}}$ data, there is a discrepancy particularly in the forward region, where the lowest values of $x_{\gamma}^{\mathrm{OBS}}$ are probed. This discrepancy shows no sign of dying away with increasing $E_{T}^{\text {Jet }}$ even though hadronization effects are estimated to be small at these values.

The potential of such data may be illustrated by assuming LO QCD \& MC models, and estimating a "parton level cross section", from which an effective parton density can be extracted. This exercise has been performed by H1, both in the case of jets and charged particles and the result is shown in figure 6a. The rise in the gluon distribution, which will drive the rise in $F_{2}^{\gamma}$ at lower $x$, is clearly in the region of HERA sensitivity, although the model dependence in the data is now large.

The physics of hard scattering in photon-proton collisions is far from trivial. The photon remnant is an interesting feature of the final state. Some of its properties have been measured by ZEUS [19]. In particular it was measured to have an average 

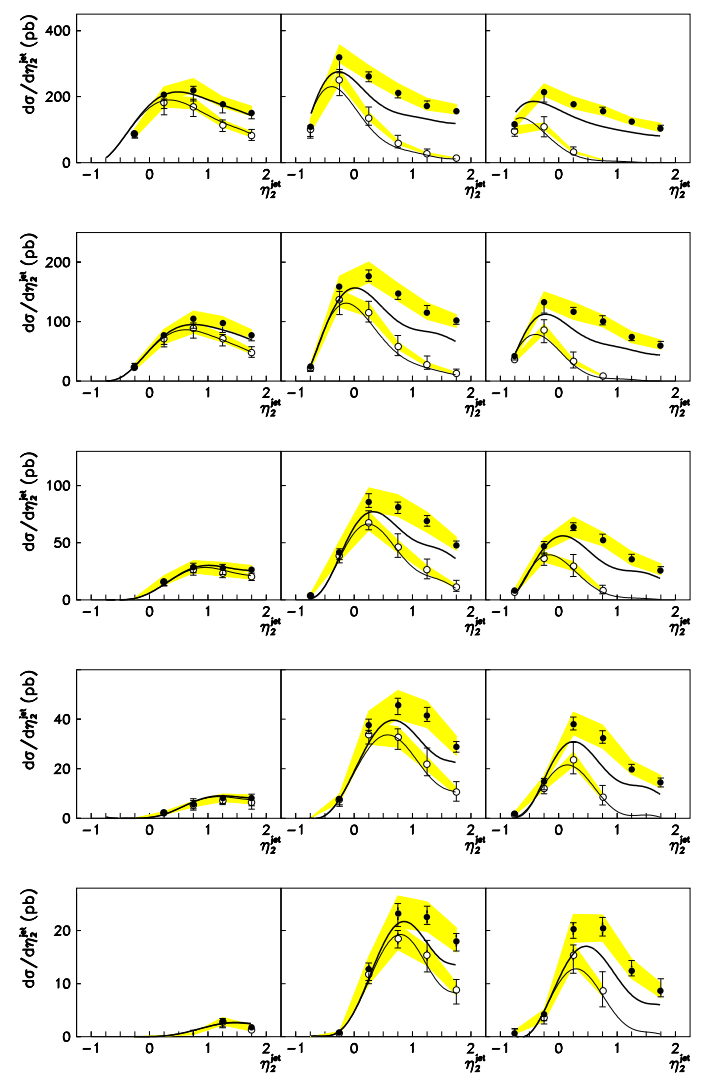

Figure 5: Dijet cross sections at HERA.

transverse momentum $p_{T}=2.1 \pm 0.2 \mathrm{GeV}$ w.r.t. photon direction. These measurement have now been extended by H1, measuring the remnant as a function of $E_{T}^{\mathrm{Jet}}$ for photoproduction and for virtual photons $\left(1.4<\mathrm{P}^{2}<25 \mathrm{GeV}^{2}\right)$. These results, shown in figure $6 \mathrm{~b}$, are consistent ZEUS result. Importantly, the behaviour of the photon remnant is critical for the $x$ resolution at LEP, since it determines how much hadronic energy escapes down the beam-pipe. HERWIG (shown in the figure) does a reasonable job, and such distributions are used to constrain the models employed at LEP, thus reducing the systematic errors.

The fact that the photon has a dual nature - behaving either as a hadron or a point-like particle - allows several interesting QCD studies to be made. A recent measurement is that of the three-jet distributions. The QCD dynamics of the three jet system is sensitive to the colour of the incoming partons. In figure $7, \theta_{3}$, the angle between the highest energy jet and the proton beam direction (defined as in [6]), is shown, and compared to $\mathcal{O}\left(\alpha \alpha_{s}^{2}\right)$ QCD and to LO MC simulation, in which the third 

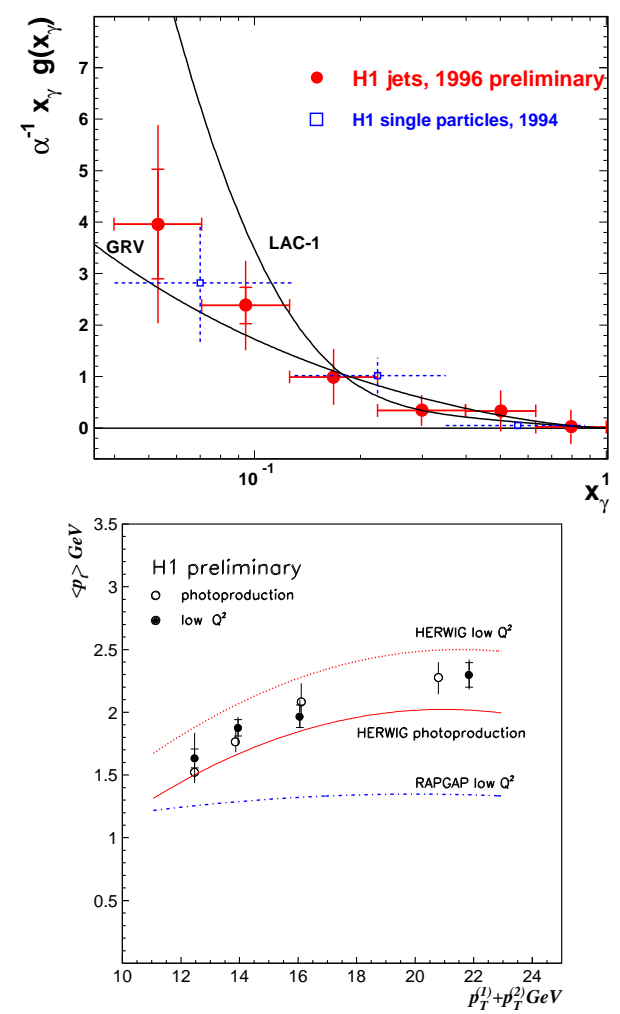

Figure 6: (a) Effective photon PDF [4], (b) Transverse energy of the photon [20] remnant.

jet comes from the parton shower. There is a change in shape of the distribution as $x_{\gamma}^{\text {OBS }}$ increases and the mix of incoming resolved and direct photons changes.

Charged particle distributions are also sensitive to photon PDFs. They also require non-perturbative input in the form of a fragmentation function, but once this is taken into account, there are no hadronization uncertainties as such. However, there is still sensitivity to the modelling of the underlying event and the choice of hard scale (see figure 8).

In addition to these processes there are prompt photon data [21], as well as measurements of jet shapes and sub-jets at HERA and LEP [22], all of which have the power to reduce the uncertainties in the final state and in the theory, if taken together. Given this enormous data set, of increasing accuracy and scope, the time is right to do a serious QCD fit to the HERA and LEP data!

In the final sections of this presentation is describe briefly two areas of photon structure studies. Both are relatively new, and both offer new and possibly simpler ways to investigate the underlying physics. 

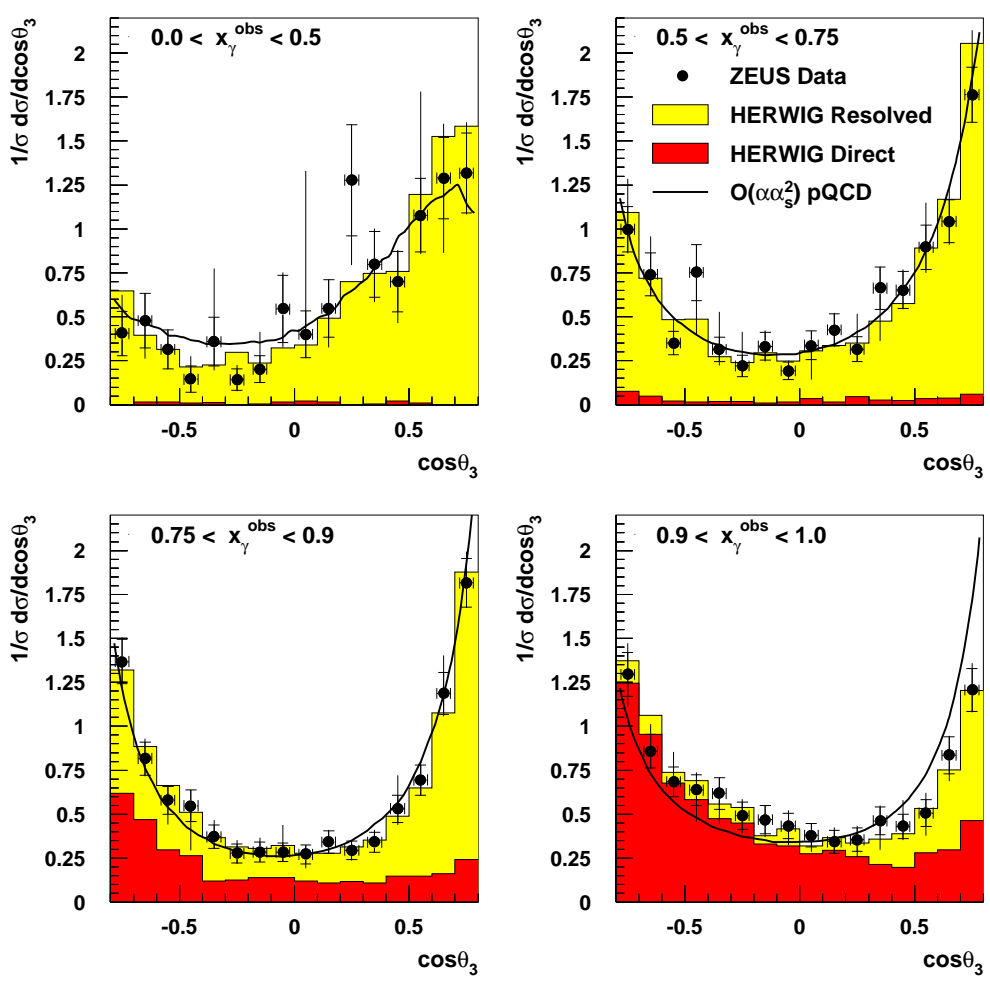

Figure 7: Three jet cross sections at HERA. LO resolved photon processes are shown as red, and LO direct as yellow.

\section{Charm and the Real Photon}

Charm photoproduction has been measured at both HERA and LEP [7, 8]. If it is assumed that the charm mass is sufficiently high that perturbative QCD is applicable, then the 'charm content' of the photon is expected to be a totally perturbatively calculable parton distribution. In fact if the factorization scale is taken lower than the charm mass, charm production takes place entirely within the hard process and there is no charm content to the photon. The data are beginning to address whether this picture is sufficient. Charm is typically tagged in the $D^{*}$ channel and recent measurements of $D^{*}$ cross sections are shown in figure 9 .

The agreement with theory is reasonable. However, the theory lies somewhat below the data in the forward (proton) direction in the HERA data. If jets are measured, $x_{\gamma}^{\mathrm{OBS}}$ can be calculated and we can begin to examine the production mechanism in more detail. The $x_{\gamma}^{\text {OBS }}$ distributions from ZEUS and OPAL are shown in figure 10 where at least one jet contains a $D^{*}$.

Comparison to the LO Monte Carlo shows that direct and resolved processes are both needed. This is still true even when the data is compared to NLO QCD [11]. The 

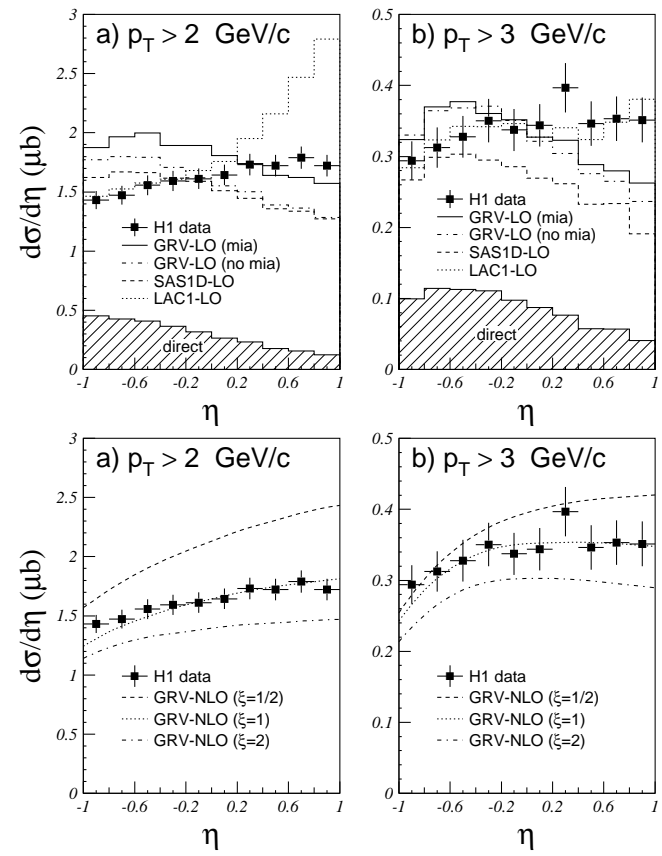

Figure 8: Charged particle distributions [4]. The curves marked "mia" or "no mia" have multiparton interactions turned on or off respectively.

photon PDF used in the calculation contains no charm, but events can be generated at low $x_{\gamma}^{\mathrm{OBS}}$ where a third jet plays the role of the photon remnant. The resolved processes are suppressed relative to direct in comparison to the non charm-tagged case, but the cross section is still significant at low $x_{\gamma}^{\mathrm{OBS}}$. Beauty in photoproduction has also been seen now at both HERA and LEP [24].

\section{$5 \quad$ Virtual Photon Structure?}

All current studies of the structure of the photon in fact study photons with a finite (if small) virtuality. Nevertheless, there has been a marked discontinuity in the terminology and methodology used to describe the photon as a propagator (in electron-proton DIS for example) and the photon as a target. This is despite the fact the ep "DIS" experiments extend well down below $1 \mathrm{GeV}$ in $\mathrm{Q}^{2}$, where the term "deep" inelastic scattering is arguably inappropriate, and also despite the fact that, as seen in the QED structure results (figure 2), the effect of target photon virtualities is significant even in so-called "photoproduction" experiments.

This situation is changing, and a significant amount of attention is now being paid 
ZEUS $1996+97$
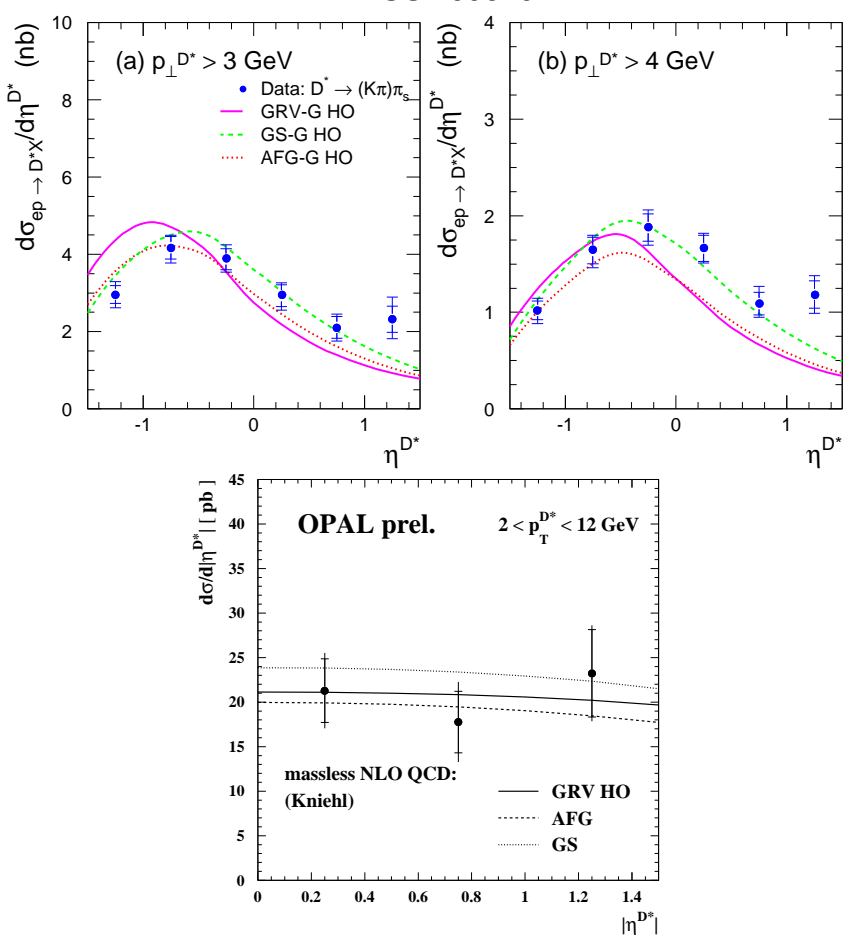

Figure 9: Inclusive $D^{*}$ photoproduction cross sections from HERA and LEP [7]. The data are compared to [23]

both by theory and experiment to the fact that there must be a continuum between $\mathrm{P}^{2}=0$ and $\mathrm{P}^{2} \approx \mathrm{Q}^{2}$. There exist some expectations as to how the transition between the two might take place. With respect to direct photon processes, the expectation is that the perturbative part of the photon structure will fall like $\ln \left(\mathrm{Q}^{2} / \mathrm{P}^{2}\right)$ whilst the non-perturbative ("Vector Meson") part should fall something like $m_{v}^{2} /\left(m_{v}^{2}+\right.$ $\left.\mathrm{P}^{2}\right)$, where $m_{v}$ is a the mass of the vector meson state into which the photon may fluctuate. When the photon virtuality gets large, but remains much less than the probing scale (which may be set by high $E_{T}^{\text {Jet }}$ jets, for example), the non-perturbative part vanishes and once more we obtain a perturbatively calculable parton distribution, which suggests possibilities for the measurement of $\alpha_{s}$ and an improved understanding of QCD radiation and hadronic structure.

Look first at the HERA data, some of which is shown in figure 11 [25]. In figure 11a, $\mathrm{H} 1$ dijet data $d \sigma / d x_{\gamma}^{\mathrm{OBS}}$ is shown in a grid in which the probing scale $\left(\approx\left(E_{T}^{\mathrm{Jet}}\right)^{2}\right)$ increases from left to right, whilst the target scale (the photon virtuality $\mathrm{P}^{2}$, here labelled $\mathrm{Q}^{2}$ according to the convention at HERA). Concentrate for instance on the second row. The target photon virtuality is in the range $3.5 \mathrm{GeV}^{2}<\mathrm{P}^{2}<$ 
$8.0 \mathrm{GeV}^{2}$, certainly far from zero, and yet the population of events at low $x_{\gamma}^{\mathrm{OBS}}$ is significant. The LO QCD plus parton showers simulation can only successfully model the distribution by appealing to a virtual photon structure ansatz [26] in which the expectations above are implemented. A similar effect is observed in the ZEUS measurement (figure 11b) where the ratio of the high to low $x_{\gamma}^{\text {OBS }}$ cross sections is plotted, now extending all the way down to the "almost real" photons previously studied. The ratio falls rapidly. However, at the lowest target virtualities it is higher than the expectations shown, and even by $4 \mathrm{GeV}^{2}$ it remains higher than the expectation of a DIS Monte Carlo which contains no photon structure. The blue line is a "straw man" model in which the GRV real photon PDF has been used in virtual photons without modification. It is not expected to be valid here, but the fact that it is completely flat demonstrates graphically that the observed fall in the data is genuinely due to suppression of the photon structure, and not to any subtle phase space effect. The Schuler and Sjöstrand parton distribution function (red curve) contains a model for the virtual photon structure which a suppression with increasing virtuality. It is interesting to note that whilst both curves lie below the data at the lowest virtuality, the SaS prediction falls more slowly than the data and thus there is agreement at the higher virtualities. The discrepancy at low virtuality (and at jets $E_{T}^{\mathrm{Jet}}$ of around $6 \mathrm{GeV}$, where these data lie) has been observed before and attributed to the effect of a so-called "underlying event", possibly generated by multiparton interactions. Such effects are not included in the curves shown here. Since models of underlying events rely upon the hadronic nature of the photon, it is natural that any discrepancy due to them should fall as the hadronic component is suppressed.

Measurements of virtual photon structure have also been made in $e^{+} e^{-}$experiments [27]. Both the early PLUTO data and the more recent LEP data are consistent with being flat with $\mathrm{P}^{2}$, but are also consistent with the expected fall.

There is also a relation between virtual photon structure and low- $x$ physics: Two virtual photons in collision is as near as we are likely to get to a "golden" process in which the total cross section is expected to be governed by a pomeron (multi-gluon colour-singlet exchange) calculable in perturbative QCD according the the BFKL [28] resummation. Such processes have been measured at LEP. Both leptons are tagged and so there is a good measurement of both photon virtualities. In contrast to the previous situations, these virtualities are now selected to be of comparable size. The idea is that there should be a large evolution in $x$ (actually in rapidity), and that the high virtualities mean that non-perturbative effects should be small. These are the conditions in which the BFKL resummation of $\ln (1 / x)$ terms should be applicable. Although the measurements so far are above the naive two-gluon exchange calculation, they are also consistent with the model encoded in PHOJET. There is a large uncertainty in the actual prediction of BFKL, and a conclusive test has yet to be made. This field is developing rapidly in both theory and experiment. 


\section{Summary}

This is a field in which lots of new data has appeared over the past two years, and more is expected soon. The new results from LEP and HERA demonstrate the improvements being made in understanding of hadronic initial and final states, using the photon as a flexible test case. This has been made possible by the emergence of several new theoretical tools, including better general purpose simulations, implementations of virtual photon PDFs, and NLO QCD calculations which allow realistic kinematic cuts to be applied. Such efforts are proving critical in extracting fundamental physics from the data.

The final word from LEP, LEP2 and pre-upgrade HERA will be a series of measurements with much reduced systematic uncertainties over a very wide kinematic range. The data and theoretical tools are now in place for a comprehensive analysis of photon structure along the lines of those carried out for the proton. To challenge our ideas about QCD structure in a second hadron-like object, with the expected differences and similarities described in this review, is a great opportunity and promises to set the essential technology of reliable QCD calculations on a significantly firmer footing.

In the slightly longer term future, charm and beauty photoproduction will be a boom area at HERA after the upgrade, as both the luminosity and the ability of the detectors to tag heavy flavours should increase markedly.

I believe that the curious nature of the photon, in which by making judicious selection we can turn on or off its "hadronic structure" is an enormously valuable tool for understanding hadronic initial and final states in general, a topic of increasing importance across the breadth of particle physics.

I am very glad to acknowledge to all the hard work involved on the LEP and HERA experiments, as well as the clearly written papers for EPS and particularly all the lively discussions with many people at Photon99 - I'm looking forward to Photon2000. Extra thanks are due to Richard Nisius for several of the summary plots.

\section{References}

[1] OPAL Collab., Phys. Lett. B411 (1997) 387-401; Z. Phys. C61 (1994) 199; Z. Phys.C74 (1997) 33; Phys. Lett. B412 (1997) 225, E. Clay Proceedings of Photon 99, Freiburg, May 1999.

DELPHI Collaboration, Z. Phys.69 (1996) 223.

L3 Collaboration, Phys. Lett. B436 (1998) 403; Phys. Lett. B447 (1999) 147; F. Erne Proceedings of Photon 99, Freiburg, May 1999.

TOPAZ Collaboration, Phys. Lett. B332 (1994) 477. 
PLUTO Collaboration, Phys. Lett. B107 (1981) 168; Phys. Lett. B142 (1984) 111; Z. Phys. C26 (1984) 353; NPB 281 (1987) 365.

A.Böhrer, ALEPH Collab., Proceedings of Photon 99, Freiburg, May 1999.

[2] H1 Collab., Z.Phys. C70 (1996) 17-30; Phys. Lett. B314 (1993) 436.

ZEUS Collab., Eur. Phys. J.C4 (1998) 591; Phys. Lett. B384 (1996) 401-413; Eur. Phys. J.C1 (1998) 1/2, 109.

OPAL Collab., Eur.Phys.J. C10 (1999) 547-561.

[3] ZEUS Collab., Phys. Lett. B348 (1995) 665-680.

[4] H1 Collab., Eur.Phys.J. C10 (1999) 363-372 and Eur.Phys.J. C1 (1998) 97-107.

[5] ZEUS Collab., Eur.Phys.J. C11 (1999) 35-50.

J. Vossebeld, hep-ex/9909039, International Europhysics Conference on HEP, Tampere, Finland, July 15-21, 1999, to appear in the proceedings.

[6] ZEUS Collab., Phys. Lett. B443 (1998) 394-408.

[7] L3 Collab., hep-ex/9909006.

ZEUS Collab., Phys. Lett. B401 (1997) 192-206.

[8] OPAL Collab., hep-ex/9911030, submitted to Eur.Phys.J. C ZEUS Collab., Eur.Phys.J. C6 (1999) 67-83.

[9] CELLO Collab., Phys. Lett. B126B, 384-390 (1983).

DELPHI Collab., Z. Phys.C69, 223-234 (1996).

L3 Collab., Phys. Lett. B438, 363-378 (1998).

OPAL Collab., hep-ex/9902024

PLUTO Collab., Z. Phys.C27, 249-256 (1985).

TPC/2 $\gamma$ Collab., M.P. Cain et al., Phys. Lett. B147, 232-236 (1984).

[10] J. Forshaw, M. Seymour, Proceedings of Photon 95, 3-9, (Sheffield) World Scientific, Ed: D.Miller, S.Cartwright, V.Khoze.

[11] S. Frixione, Nucl. Phys. B507 (1997) 295.

[12] B.W. Harris and J.F. Owens, Phys. Rev. D56 (1997) 4007.

M. Klasen and G. Kramer, Z. Phys. C76 (1997) 67, M. Klasen, T. Kleinwort and G. Kramer, Eur. Phys. J.1 (1998).

B. Pötter, Comp. Phys. Comm. 119 (1999) 45

[13] G. Marchesini et al., Comp. Phys. Comm.67 (1992) 465 and hep-hp/9607393. H.U. Bengtsson and T. Sjöstrand, Comp. Phys. Comm.46 (1987) 43 and T. Sjöstrand, Comp. Phys. Comm.82 (1994) 74.

R. Engel, Z. Phys. C66 (1995) 203. 
[14] Proceedings of the HERA Monte Carlo Workshop 1998/99, DESY, Ed: A.T.Doyle, G.Grindhammer, G.Ingelman, H.Jung http://www.desy.de/ heramc/proceedings/

A. Finch et al, J.Phys. G24, (1998) 457.

A. Finch (LEP experiments) Proceedings of Photon 99, Freiburg, May 1999.

[15] M. Glück et al., Phys. Rev. D46 (1992) 1973.

[16] V.N. Gribov and L.N. Lipatov, Sov. J. Nucl. Phys. 15 (1972) 438 and 675;

Yu.L. Dokshitzer, Sov. Phys. JETP 46 (1977) 641;

G. Altarelli and G. Parisi, Nucl. Phys. B126 (1977) 297.

[17] R. Nisius, hep-ex/9907012, Invited talk given at Photon 99, Freiburg, May 1999.

[18] P. Aurenche et al., Z. Phys.C64 (1994) 621.

[19] ZEUS Collab., Phys.Lett. B354 (1995) 163-177.

[20] Alice Valkarova (H1 Collab.), Proceedings of Photon 99, Freiburg, May 1999.

[21] ZEUS Collab., Phys. Lett. B413 (1997) 201-216.

[22] ZEUS Collab., Eur.Phys.J. C2 (1998) 61-75.

Sabine Wölfle (ZEUS Collab.) Proceedings of Photon 99, Freiburg, May 1999.

[23] B.A.Kniehl et al., Z. Phys. C76 (1997) 689;

J.Binnewies et al., hep-ph/9712482, Phys. Rev. D58 (1998) 014014.

[24] H1 Collab. Phys. Lett. B467 (1999) 156.

O. Deppe, (ZEUS Collab.), Proceedings of Photon 99, Freiburg, May 1999.

R. McNeil, (L3 Collab.), Proceedings of Photon 99, Freiburg, May 1999.

[25] H1 Collab., Phys. Lett. B415 (1997) 418-434 and hep-ex/9812024, submitted to Eur. Phys. J. C.

Dorian Kcira, ZEUS Collab., Proceedings of Photon 99, Freiburg, May 1999.

[26] M. Drees, R. Godbole, Phys. Rev. D50 (1994) 3124.

[27] PLUTO collab., Phys. Lett. 142 (1984) 119.

P. Achard (L3 Collab.) Proceedings of Photon 99, Freiburg, May 1999.

M. Przybycien (OPAL Collab.) Proceedings of Photon 99, Freiburg, May 1999.

[28] I. Balitsky and L.N. Lipatov, Sov. J. Nucl. Phys. 28 (1978) 822. 

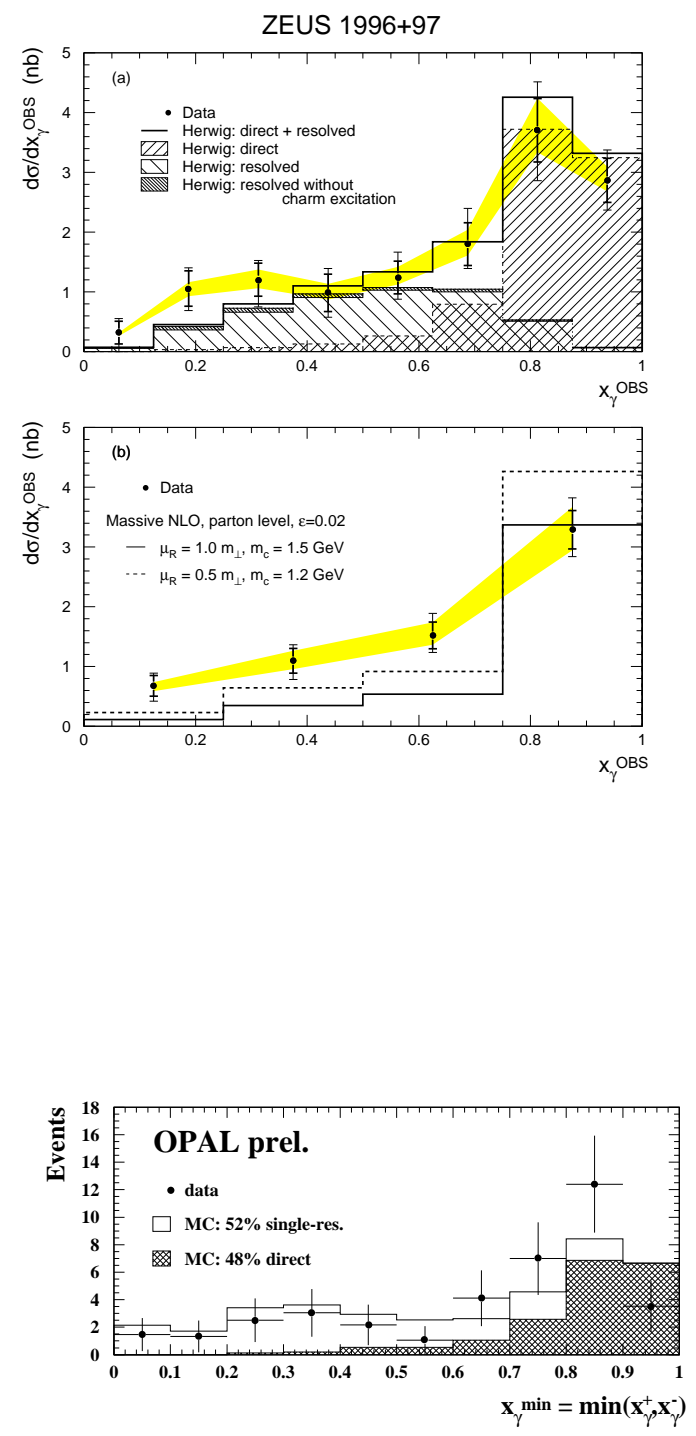

Figure 10: ZEUS cross section and OPAL event distribution as a function of $x_{\gamma}^{\text {OBS }}$ from charm-tagged dijet events [8]. 


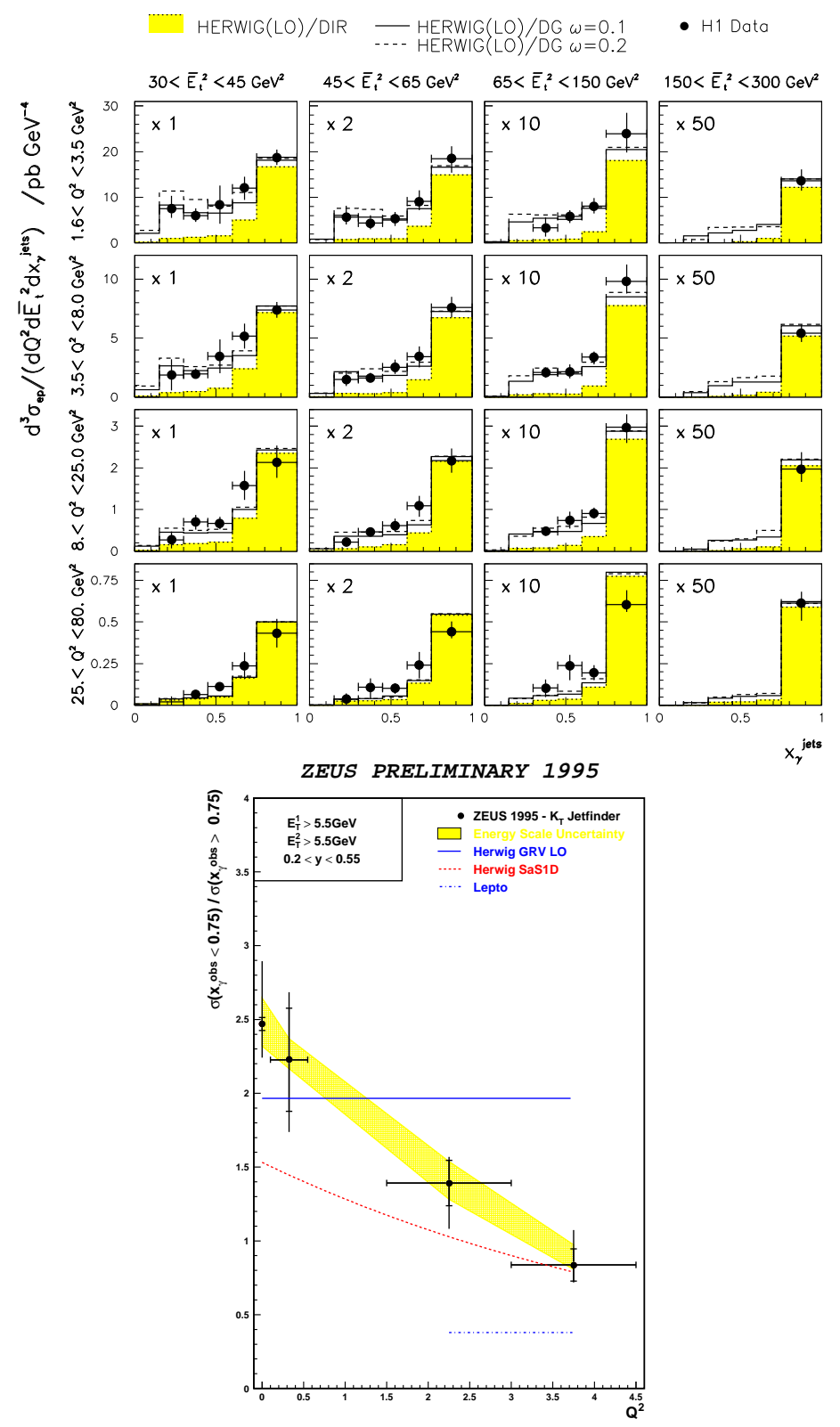

Figure 11: Virtual photons resolved. 